\title{
A new approach to the hourly mean computation problem when dealing with missing data
}

\author{
S. Marsal and J. J. Curto \\ Observatori de l'Ebre. CSIC — Universitat Ramon Llull Carretera de l'Observatori, 8. 43520 Roquetes, Spain
}

(Received November 7, 2008; Revised May 8, 2009; Accepted May 14, 2009; Online published October 19, 2009)

\begin{abstract}
Geomagnetic observatory records are unavoidably affected by primary data interruptions which, in turn, may have possible effects on the accuracy of the definitive data derived from them. One of the products most widely used by the scientific community is the mean hourly values, immediately obtained from the primary minute values of the geomagnetic field. Although some precepts have already been proposed and used, a definitive criterion regarding the procedure to follow when dealing with missing data has not yet been established. This could be seen in the last IAGA meetings and workshops, where several constructive opinions were put forward in this respect. The present discussion is devoted to analyzing the effects that different amounts of missing data have upon the accuracy of the means, a necessary step before establishing a definitive rule as to how to deal with these situations. In this statistical approach, we propose a new criterion based on the relative value of the root mean square error (between actual and computed means) with respect to the natural magnetic field variations of the original hourly interval.
\end{abstract}

Key words: Mean hourly values, uncertainty, geomagnetism, accuracy, confidence level, missing data, data processing, statistics.

\section{Introduction}

The mean hourly values of the Earth's magnetic field elements as recorded by ground-based observatories are used in a number of studies dealing with medium term variations, such as those related to the $S_{\mathrm{q}}$ system of currents (Green, 1972; Torta et al., 1997) or the EEJ (Rangarajan, 1982). They are also employed in magnetic field modelling (Walker et al., 1997), indexing (Martini and Mursula, 2006; Svalgaard and Cliver, 2007), and even in the study of $S_{\mathrm{q}}$ trends (Le Mouël et al., 2005; Torta et al., 2008). They provide representative values of the magnetic field within the hours of interest, which are useful when dealing with intermediate timescale magnetic features. Regarding the cases of long and short timescales, high time resolutions are required when studying shorter timescale magnetic phenomena such as Pi2 (1-second data) or Sfe (1-minute data), while low time resolutions are used in the study of longer timescale phenomena such as magnetic jerks (monthly values) or secular variation (annual values).

A moderate time resolution also implies that high accuracy in the magnetic field magnitude is simply not required for many purposes. Hence we are faced with the question as to what the required level of accuracy for the hourly means is. For practical reasons the answer to this question should be as general as possible, although it probably depends on several factors, such as the type of study carried out by each particular data user. Likewise, it seems clear that the degree of magnetic field disturbance in the relevant hourly inter-

Copyright (c) The Society of Geomagnetism and Earth, Planetary and Space Sciences (SGEPSS); The Seismological Society of Japan; The Volcanological Society of Japan; The Geodetic Society of Japan; The Japanese Society for Planetary Sciences; TERRAPUB val also plays an important role. Suppose, for instance, that the magnetic field variation during a given disturbed hourly interval is $500 \mathrm{nT}$. For many purposes it will probably be meaningless to provide a mean hourly value (MHV) with an accuracy of $1 \mathrm{nT}$, since it will not add substantial information to our study. An accuracy of $50 \mathrm{nT}$ may well suffice. On the contrary, an hourly variation of $5 \mathrm{nT}$ will certainly require a more accurate mean to distinguish the fine natural variations we may be interested in, such as those related to the $S_{\mathrm{q}}$. Following this reasoning, we hereafter suggest establishing a criterion based on the standard deviation of the magnetic field variations computed from the (original) minute values in the hour, rather than establishing one definite, clear-cut value for accuracy. Thus, the question to be addressed to MHV data users is: What fraction of the standard deviation represents the required MHV accuracy? The answer to this question requires a deep analysis of the diverse uses of MHVs that we, as data providers, will not attempt to undertake here.

The problem of missing data within the hourly intervals is related to this issue. An absence of data is frequently a consequence of acquisition problems, or is derived from the data post-processing itself. There are several opinions regarding the most adequate procedure to follow in the presence of data gaps, as shown in the last IAGA meetings and workshops. One of the fundamental questions to be addressed is: How many minute data can be lost in one hour without the hourly mean losing significance? This is directly related to the question addressed in the previous paragraph regarding the required accuracy of the MHVs. If the reported mean stays within the required accuracy despite a given number of missing data, then this number is 
Table 1. Geomagnetic and geographic coordinates of the observatories used in this study.

\begin{tabular}{lcccc}
\hline & $\begin{array}{c}\text { Geomagnetic latitude } \\
\end{array}$ & Geomagnetic longitude & Geographic latitude & Geographic longitude \\
& $\left({ }^{\circ} \mathrm{N}\right)$ & $\left({ }^{\circ} \mathrm{E}\right)$ & 64.9 & 212.2 \\
\hline College, CMO & 65.4 & 261.7 & 40.1 & 254.8 \\
Boulder, BOU & 48.4 & 320.6 & 18.1 & 293.8 \\
San Juan, SJG & 28.3 & 6.1 & 40.8 & 0.5 \\
Ebre, EBR & 43.2 & 81.3 & -62.7 & 299.6 \\
Livingston Is., LIV & -52.6 & 8.6 & & \\
\hline
\end{tabular}

considered permissible. Of course, this question cannot be answered a posteriori, since we would not be able to calculate the actual or true mean (i.e., with the complete data set). In this sense, the aim of the exhaustive works of Mandea (2002) and Schott and Linthe (2007) was to establish a rule capable of, a priori, answering the following question: Given a tolerable number of missing data, to what extent does the estimated mean depart from the actual mean? In their studies, these authors find major dependences on the latitude of the analyzed observatory and on the underlying magnetic field activity, which leave questions unanswered regarding the MHV computation problem.

Our approach differs slightly in that, given a number of missing data, we are interested in finding out by what fraction of the standard deviation (of the original data) the estimated mean departs from the actual mean. For now, let us refer to this concept as relative accuracy. For the same reasons as those set out above, we think this is a more physically significant parameter than an absolute lower limit for the accuracy (e.g., $1 \mathrm{nT}$ ). Obviously, there are so many possibilities of different distributions of gaps within the hour, and the level of magnetic activity of the lost data, that the outcome will be a distribution of probability. If the aforementioned required relative accuracy were wellestablished, the question of missing data could be addressed in the following way: What is the maximum number of missing data we can permit, while being reasonably confident that the (pre-established) required relative accuracy is still achieved? We will situate this confidence level at $95 \%$ probability; in other words, we will say we are reasonably confident that the required limit of relative accuracy will not be surpassed if its probability of occurrence is less than 5\%. Because it is useful for error propagation, we are also interested here in the relative standard deviation between the estimated and actual means.

Given that we will not assume any pre-established relative level of accuracy for the MHVs, in the following sections we will invert the previous question and try to provide an answer to the equivalent one: What is the relative accuracy of the estimated MHV when dealing with different numbers of missing data, whether they be $2,10,35,50 \ldots$ ? Once the required relative accuracy has been determined (by others), the answer to the last question will immediately allow us to set the maximum number of missing data.

\section{Procedure and Results}

The answer to the last question demands a statistical analysis based on real data. Given a complete test hour (i.e., with no missing data), how does the mean respond to the (artificial) extraction of minute values? As pointed out be- fore, it will depend on the way these data are extracted. Among other interesting points, the work from Schott and Linthe (2007) performs a complete analysis on the real distribution of data and gap segments within the hourly intervals of a given station (PAF). It concludes that the probability of having more than one gap segment per hour is relatively small (of the order of $10 \%$ or less); likewise, our calculations for the Ebro Observatory for the period 20012005 yield a $94 \%$ probability for a 1 gap segment and $6 \%$ for 2 gap segments, with cases of 3 or more segments being virtually non-existent. In fact, Mandea (2002) takes as a working hypothesis a single gap segment of variable length.

In this work we will proceed by following two scenarios: in the first one data are extracted randomly to simulate randomly scattered missing data, while in the second one data are extracted as a continuum to simulate one single gap. As a rule, and assuming a fixed number of missing data, the greater the number of gap segments within the hourly interval, the closer both the estimated and actual means are. We can see this intuitively as the information lost with a short gap segment can, to a certain extent, be interpolated by the neighbouring minutes. Hence, although the first case is generally unlikely, it will be useful to show how different the results from both situations are. Finally, an upper limit is established for the uncertainty of the estimated mean when dealing with a realistic distribution of missing data, i.e., considering the adequate proportion of gap segments.

The real data used in this study are taken from geomagnetic stations at different latitudes: College (CMO) at high latitude, Boulder (BOU) at mid-latitude, and San Juan (SJG) at low latitude, for which the $X$ magnetic element was analyzed. Furthermore, we have made use of the $H$ element of the observatories run by the authors, namely the Ebro (EBR) and Livingston Island (LIV) ones, see Table 1. The use of these magnetic elements is due to their greater impact in indexing and modelling practices. The data have also been selected in such a way that they cover different magnetic activity levels: active, A (local $K$-index $=8$ ), moderate, $\mathrm{M}(K=5)$ and quiet intervals, $\mathrm{Q}(K=0)$. 24 hours have been used for a given observatory and disturbance level, so that a total of 360 (5 stations $\times 3$ activity levels $\times 24 \mathrm{~h}$ ) hourly intervals have been analyzed.

Our immediate objective is to obtain the uncertainty of the mean computed when possessing $60-g$ data points, where $g$ is the number of missing data. We will proceed by following a Type A evaluation, as defined in the Guide to the Expression of Uncertainty in Measurement ('GUM', ISO, 1993), in accordance with the subsequent steps:

(1) From our set of 360 hourly intervals we choose one 


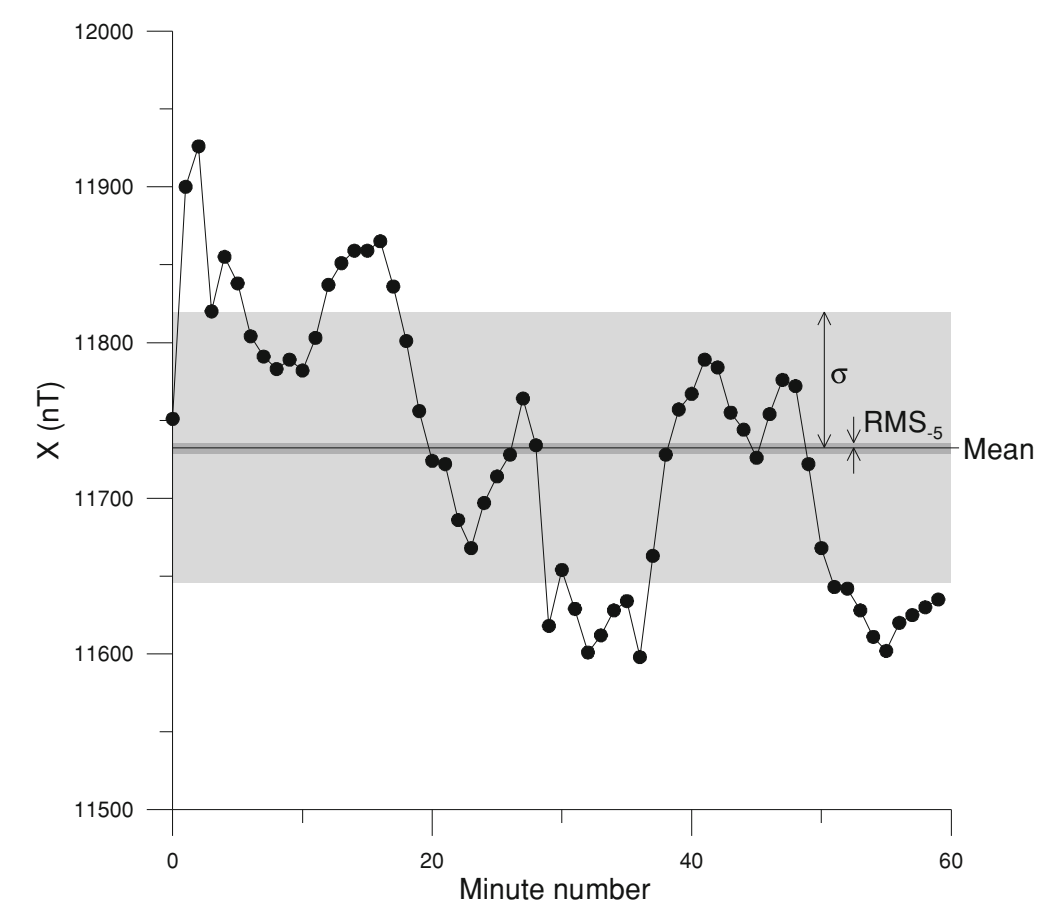

Fig. 1. $X$-component minute values of the disturbed hourly interval recorded at the CMO observatory on January 17th, 2005, 09:00-10:00 UT. The standard deviation $\sigma$ and the RMS value obtained after random extraction of 5 data points are also displayed.

complete hourly interval for testing.

(2) $g$ minute values are deleted from the selected hourly interval and the new (or estimated) mean $\langle x\rangle_{-g}$ is computed

(3) The actual mean $\langle x\rangle_{0}$ (i.e., with no deletions) is subtracted from $\langle x\rangle_{-g}$.

(4) The preceding steps are repeated for different extraction combinations of $g$ data points within the same hourly interval. The values $\langle x\rangle_{-g}^{i}-\langle x\rangle_{0}$ are thus obtained, where the $i$ index denotes each particular choice of extraction.

(5) The root mean square of these differences is computed by means of Eq. (1):

$$
\mathrm{RMS}_{-g}=\sqrt{\frac{\sum_{i=1}^{l}\left(\langle x\rangle_{-g}^{i}-\langle x\rangle_{0}\right)^{2}}{l-1}}
$$

(6) The 95th percentile of the distribution of the differences obtained in step 4 is computed, giving rise to the so-called 'uncertainty at the level of confidence of 95\%, $U_{-g}^{95}$ (see ISO, 1993).

(7) The quotients $\mathrm{RMS}_{-g} / \sigma$ and $U_{-g}^{95} / \sigma$ are computed, where $\sigma$ is the standard deviation of the geomagnetic data in the original hourly interval and gives an idea of the natural magnetic field activity.

We can interpret $\mathrm{RMS}_{-g}$ as a representative value of the deviation of the new mean (after the extraction of $g$ data minutes) from the actual mean. Note that the RMS value obtained from Eq. (1) is not stricto sensu the standard deviation of the distribution of the differences as, in general, the mean of the different $\langle x\rangle_{-g}^{i}$ (with respect to $i$ ) is different from $\langle x\rangle_{0}$. Our aim is to study the statistical response of ap- plying steps 1 to 7 (with different $g$ values) to the different 360 hourly intervals.

\subsection{Random extraction}

In this case, the data in our test hourly interval are eliminated in a random way, so that for each number $g$ of missing data, 1000 different extraction combinations are made (i.e., $l=1000$ in Eq. (1)). For example, for $g=5$ a first choice of deletions $(l=1)$ might correspond to minutes 12 , $18,31,44$ and 57 , for $l=2$ the deleted minutes might be $00,07,23,24$ and 44, and so on. A series of tests indicate that $l=1000$ provides a sample large enough to obtain significant results. This will give rise to a distribution of the differences between estimated and actual means, which we will show to be well-approximated by a normal distribution centred at zero. This fact will permit us to note that (only for the case of random extraction) the RMS $/ \sigma$ value is effectively the same as the relative standard deviation of the distribution. After many observations we will arrive at the important result that, whatever the hourly interval we consider (regardless of the latitude or activity level), this relative standard deviation is constant for a given number of missing data. With these results, we will infer the $95 \%$ confidence level to be the value given by twice the RMS $/ \sigma$. Finally, we will provide a plain statistical justification that approximates the results we find.

Figure 1 shows the minute values of the active (A) hourly interval corresponding to January 17th, 2005, 09:0010:00 UT as recorded at the CMO observatory. The differences of the means after randomly extracting 5 data minutes are distributed as shown in Fig. 2. Note that Fig. 1 also displays $\sigma$, the standard deviation of the 60 minute values of the geomagnetic field, and $\mathrm{RMS}_{-5}$ obtained from the distribution of Fig. 2.

The middle row of Table 2 shows the $\mathrm{RMS}_{-g}$ values en- 


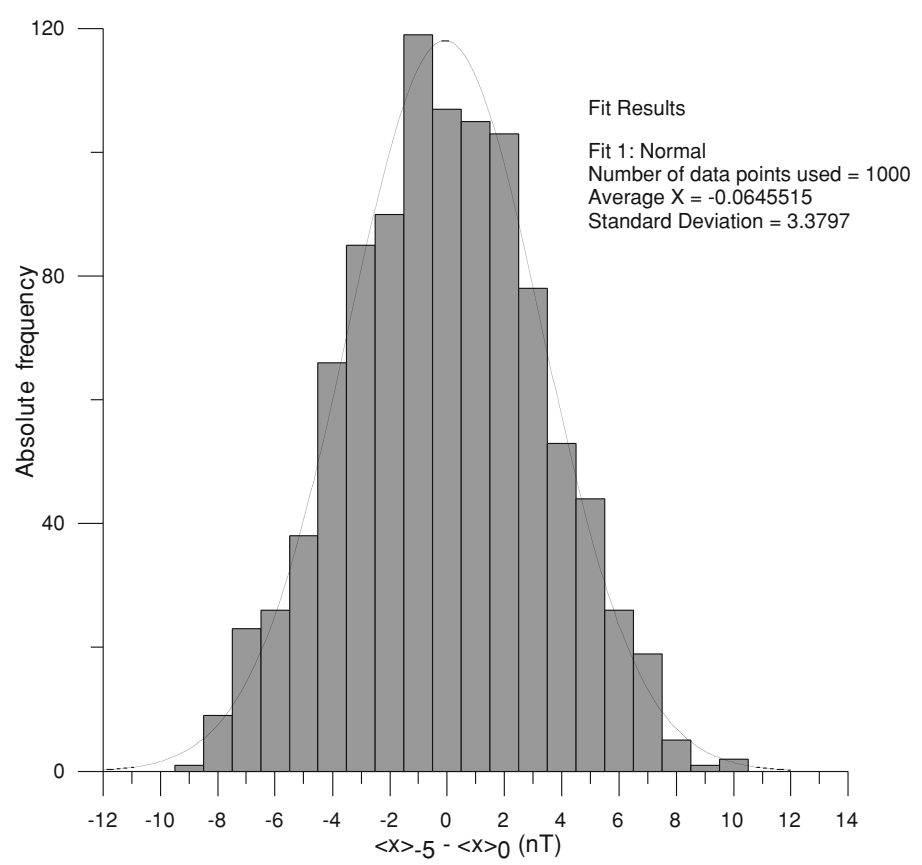

Fig. 2. Histogram showing the distribution of the 1000 differences of the means obtained after random extraction of 5 data points for a high-latitude observatory (CMO) and high activity level (A); hourly interval: January 17th, 2005, 09:00-10:00 UT.

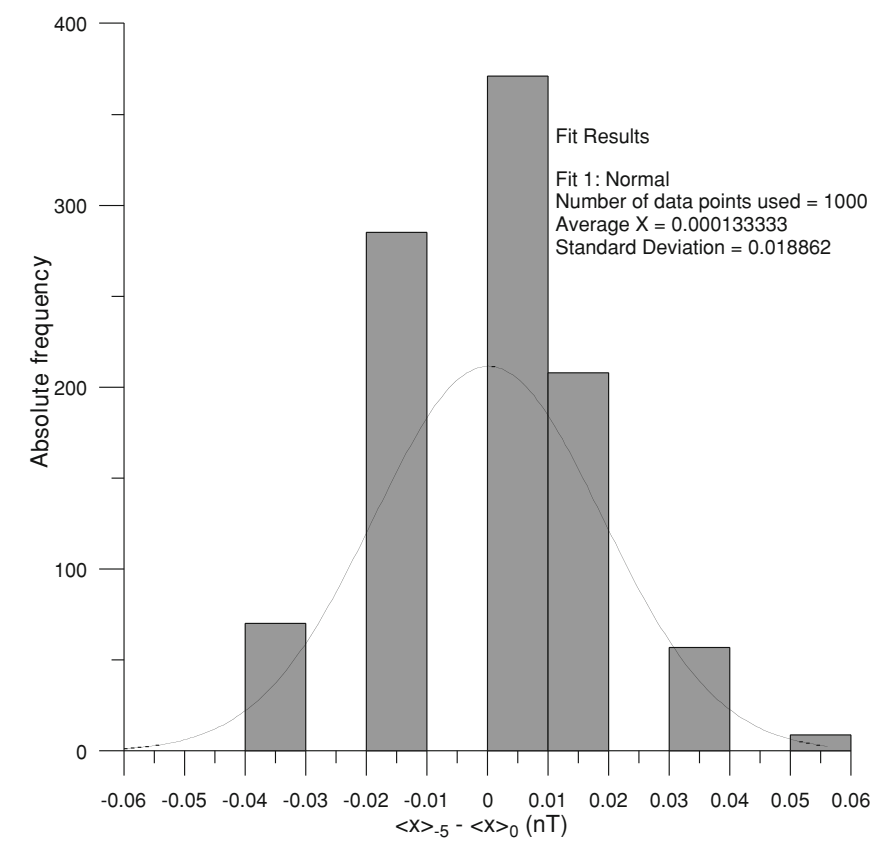

Fig. 3. Distribution of the 1000 means after random extraction of 5 data points for the hourly interval 00:00-01:00, Nov 16th, 2005, for SJG Q (quiet). Note that the Gaussian curve does not fit the observed data well. The striped structure of the distribution is an effect of the limited resolution of the primary minute data applied to such an extremely quiet interval.

countered for the hourly interval of our example when applying different values of $g$. It is worth mentioning that the RMS value as computed from Eq. (1) is $3.38 \mathrm{nT}$ (Table 2), which coincides with the standard deviation of the distribution of the estimated means (fit results in Fig. 2). This is not only the case for the hourly interval of Fig. 2 but, rather, it is a general result observed whenever dealing with random extraction, due to the fact that the distribution is well centred at $\langle x\rangle_{0}\left(\left\langle\langle x\rangle_{-5}^{i}-\langle x\rangle_{0}\right\rangle=-0.06 \mathrm{nT}\right.$ from Fig. 2). The reader can find another example in Fig. 3. As stated in the introduction of Section 2, this assertion is not valid in general, as we will see when dealing with the case of continuous extraction (Section 2.2).

In order to illustrate the results, we can say that after many random extractions of 5 data minutes from the hourly interval of our example, the standard deviation of the new mean with respect to the actual one is $3.38 \mathrm{nT}$ (see Table 2 or the fit results from Fig. 2). In other words, 'on average', the new mean will be $3.38 \mathrm{nT}$ away from the actual one. As observed, the distribution of Fig. 2 is well ad- 
Table 2. Root mean square (Eq. (1)) of the computed means after $g$ random deletions, and percentages with respect to $\sigma$. Application to the hourly interval of Fig. 2.

\begin{tabular}{lcccccccccccccccc}
\hline Missing data $g$ & 0 & 5 & 10 & 15 & 20 & 25 & 30 & 35 & 40 & 45 & 50 & 55 \\
\hline RMS $_{-g}(\mathrm{nT})$ & 0 & 3.38 & 5.04 & 6.47 & 7.95 & 9.42 & 11.20 & 13.13 & 15.80 & 19.55 & 24.94 & 37.24 & 86.48 \\
RMS $_{-g} / \sigma(\%)$ & 0 & 3.89 & 5.80 & 7.44 & 9.15 & 10.83 & 12.89 & 15.11 & 18.18 & 22.49 & 28.68 & 42.83 & 99.47 \\
\hline
\end{tabular}

Table 3. Relative values of the root mean square $\left(\mathrm{RMS}_{-g} / \sigma(\%)\right)$ after extraction of $g$ data points for a wide sample of stations and activity levels (A active, $\mathrm{M}$ moderate, Q quiet). Note the relatively constant value in a given column, even for hours with different $\sigma$ values.

\begin{tabular}{lcccccccccccccccccc}
\hline Missing data $g$ & 0 & 5 & 10 & 15 & 20 & 25 & 30 & 35 & 40 & 45 & 50 & 55 & 59 \\
\hline \hline $\begin{array}{l}\text { CMO A Jan 17, 2005 16:00-17:00 } \\
(\sigma=702.0 \mathrm{nT})\end{array}$ & 0 & 3.91 & 5.77 & 7.47 & 9.17 & 10.94 & 12.91 & 15.29 & 18.20 & 22.55 & 28.89 & 42.32 & 98.36 \\
\hline $\begin{array}{l}\text { CMO Q Mar 22, 2005 08:00-09:00 } \\
(\sigma=1.01 \mathrm{nT})\end{array}$ & 0 & 3.85 & 5.69 & 7.51 & 9.00 & 10.87 & 12.97 & 15.39 & 18.22 & 22.53 & 28.79 & 42.65 & 99.31 \\
\hline $\begin{array}{l}\text { BOU M Jan 12, 2005 03:00-04:00 } \\
(\sigma=18.9 \mathrm{nT})\end{array}$ & 0 & 3.86 & 5.71 & 7.53 & 9.27 & 10.94 & 13.07 & 15.37 & 18.29 & 22.49 & 28.84 & 42.71 & 99.77 \\
\hline $\begin{array}{l}\text { SJG A Oct 29, 2003 07:00-08:00 } \\
(\sigma=71.9 \mathrm{nT})\end{array}$ & 0 & 3.88 & 5.76 & 7.42 & 9.17 & 11.10 & 12.78 & 15.26 & 18.49 & 22.24 & 28.78 & 42.86 & 99.48 \\
\hline $\begin{array}{l}\text { SJG Q Nov 16, 2005 08:00-09:00 } \\
(\sigma=0.13 \mathrm{nT})\end{array}$ & 0 & 3.87 & 5.65 & 7.67 & 9.30 & 10.84 & 13.17 & 15.49 & 18.28 & 22.39 & 28.97 & 42.37 & 105.89 \\
\hline $\begin{array}{l}\text { EBR M Jan 1, 2005 18:00-19:00 } \\
(\sigma=20.38 \mathrm{nT})\end{array}$ & 0 & 3.90 & 5.77 & 7.51 & 9.06 & 10.97 & 12.95 & 15.17 & 18.13 & 22.41 & 28.87 & 42.32 & 99.76 \\
\hline $\begin{array}{l}\text { LIV Q May 14, 2004 12:00-13:00 } \\
(\sigma=2.40 \mathrm{nT})\end{array}$ & 0 & 3.88 & 5.78 & 7.49 & 9.15 & 10.96 & 12.97 & 15.27 & 18.54 & 22.07 & 28.89 & 42.75 & 100.28 \\
\hline
\end{tabular}

Table 4. Relative uncertainty at the level of confidence of $95 \%, U_{-g}^{95} / \sigma$, after random extraction of $g$ data minutes. The values are roughly twice those of Table 3 .

\begin{tabular}{lccccccccccrrrr}
\hline Missing data $g$ & 0 & 5 & 10 & 15 & 20 & 25 & 30 & 35 & 40 & 45 & 50 & 55 & 59 \\
\hline$U_{-g}^{95} / \sigma(\%)$ & 0 & 7.8 & 11.5 & 15 & 18 & 22 & 26 & 31 & 37 & 45 & 58 & 85 & $\approx 200$ \\
\hline
\end{tabular}

justed by a Gaussian curve. This agreement has also been checked for many other hourly intervals; as expected, the least favourable cases are found with quiet intervals, when $g$ is either small or large (see Fig. 3). Nevertheless, regarding the parameters we are interested in, a normal (Gaussian) distribution is still suitable even in this case. A test for this is the value of $U_{-g}^{95}$ as the observed 95th percentile of the distribution: should the data be normally distributed, the 95th percentile $(=0.0379 \mathrm{nT}$ in the case of Fig. 3, obtained after the elimination of the tail-most $5 \%$ of the distribution) must coincide with twice the value of the $\mathrm{RMS}_{-g}$ parameter $\left(2 \cdot \mathrm{RMS}_{-5}=0.0377 \mathrm{nT}\right.$, where $\mathrm{RMS}_{-5}$ is the standard deviation displayed in the fit results of Fig. 3). In summary, we infer that the normal distribution is adequate to quantify our problem, and thus (only for the case of random extraction), we will work with the hypothesis that the resultant means following random extraction are normally distributed around the actual mean, regardless of the number of missing data or activity level.

If we continue analyzing Table 2 we realize that, as expected, the root mean square increases with $g$, the number of missing data. As stated, our approach consists in normalizing the $\mathrm{RMS}_{-g}$ values with respect to the standard deviation $\sigma$ of the original data (with no deletions). If we do so and multiply by 100 to obtain the percentages, we obtain the last row of Table 2 .

A similar table has been obtained for each of the 360 analyzed hours to cover a wide spectrum of activity levels and observatory latitudes. After this, we observed that although the different $\mathrm{RMS}_{-} g$ vary, the percentages $\mathrm{RMS}_{-} \mathrm{g} / \boldsymbol{\sigma}$ are practically the same, even if we put different stations and activity levels together. Table 3 illustrates this important result with an assorted representation of latitudes and magnetic activities.

Let us refer to $\mathrm{RMS}_{-g} / \sigma$ as the 'relative standard uncertainty' of the estimated mean after the extraction of $g$ data points. However, it should be clarified that relative in this context means with respect to $\sigma$.

Assuming a normal distribution of the means (obtained after random extraction) around the actual mean, the probability that a given mean is within $2 \cdot \mathrm{RMS}_{-g} / \sigma$ is $95 \%$, which establishes our confidence level, so the values in Table 3 must be duplicated to obtain $U^{95} / \sigma$, which will be referred to as the 'relative uncertainty at the level of confidence of $95 \%$ '. In other words, after the random extraction of 5 data minutes, we can say it is reasonably unlikely that the error in the newly computed mean surpasses $2 \times 3.9 \%$ $=7.8 \%$ (see column $g=5$ in Table 3 ) of the standard deviation $\sigma$ of the original hourly data. In conclusion, if we had pre-established the required relative accuracy, the penultimate question in the introduction, regarding the maximum number of missing data acceptable, would then immediately be answered with the help of Table 4 , which is valid for any latitude and activity level.

Finally, we can provide a certain explanation for the results encountered in this section, especially in relation to the 


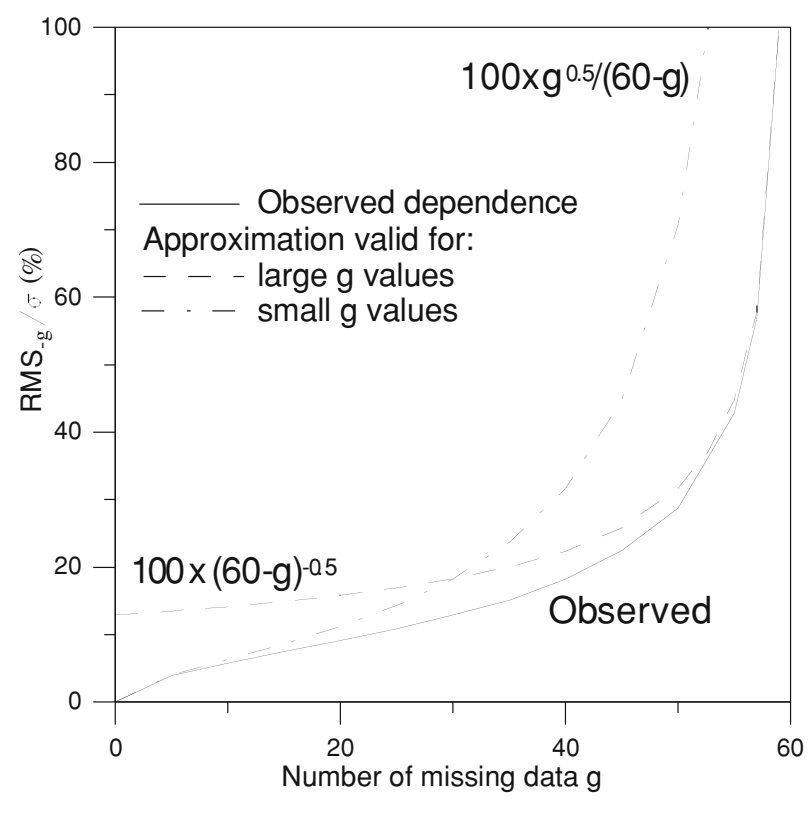

Fig. 4. Observed relative standard deviation after random extraction of $g$ data points, together with approximations for large and small $g$ values.

values of the $\mathrm{RMS}_{-g} / \sigma$ parameter (Taylor, 1982). Suppose that instead of being continuous, the magnetic field values were distributed around the actual mean $\langle x\rangle_{0}$ according to a random distribution with standard deviation $\sigma$ (the actual standard deviation of the original data). After the random elimination of 59 points from our original hourly interval, the mean deviation of the remaining 1 point with respect to the true mean is just the standard deviation $\sigma$ of the sample, so $\mathrm{RMS}_{-59} / \sigma=1$ (i.e., $100 \%$ ), which roughly coincides with the results displayed in the last column of Table 3. Likewise, we know from fundamental statistics that after the elimination of 55 data points, the standard uncertainty of the mean obtained with the remaining 5 data points is $\sigma / \sqrt{5}$, so $\mathrm{RMS}_{-55} / \sigma=1 / \sqrt{5}$ (i.e., $44.7 \%$ ), which is similar to the corresponding column with header $g=55$ in Table 3. In general, in possession of $n$ data points, the mean relative deviation with respect to the actual mean is $\mathrm{RMS}_{-g} / \sigma=1 / \sqrt{n}=1 / \sqrt{(60-g)}$. Of course, in the real case we are limited to $n=60$, where both actual and estimated means must converge, so it is expectable that the deviation with respect to the true mean decreases faster than $1 / \sqrt{n}$. At the opposite extreme (small value of $g$ ), after the deletion of $g$ data points, the best estimate of the sum of the extracted data is $g\langle x\rangle_{0}$, with a standard uncertainty of $\sigma \sqrt{g}$. This must coincide with the uncertainty of the sum of the available data, where the best estimate for this sum is $(60-g)\langle x\rangle_{0}$. The new mean equals the sum of the available data divided by the number of available data, $60-g$. Consequently, the standard uncertainty of the new mean will be $(\sigma \sqrt{g}) /(60-g)$, or $\mathrm{RMS}_{-g} / \sigma=\sqrt{g} /(60-g)$. Both approximations for small and large $g$ values are displayed in Fig. 4, together with the observed results.

\subsection{Continuous extraction}

Things are not so simple when considering the most common case - that of one single gap. The procedure we follow in this case is exactly the same as with the previous one, but instead of random extractions, we will consider a continuous extraction of variable length. The number of possible ways a single gap of length $g$ can be extracted from a given hour is reduced to $c=61-g$, which allows an analysis based on all the possible cases. Firstly, we will see that the approximation consisting in identifying RMS (Eq. (1)) with the standard deviation of the differences between estimated and actual means is, in general, no longer valid for the case of one single gap. Secondly, we will investigate the behaviour of the RMS $/ \sigma$ and $U^{95} / \sigma$ parameters and see that, unlike the previous case, they are not constant over different hourly intervals. This will hinder our objectives, and force us to adopt uncertainty intervals for the corresponding relative uncertainties themselves (i.e., $\mathrm{RMS} / \sigma$ and $U^{95} / \sigma$ ). However, we will not find a significant dependence of these two parameters on the observatory latitude or magnetic activity level, and this will allow us to set (universally) common bounds for these intervals.

Figure 5 shows the distribution of the 41 differences of the estimated means with respect to the actual (or true) mean for the case of LIV M, hourly interval: January 9th, 2004, 18:00-19:00 UT, after extracting 20 running minutes $(c=61-20=41)$.

It is clear that the histogram of Fig. 5 is far from a normal distribution. Moreover, the mean computed after extracting 20 data minutes does not coincide, on average, with the actual mean $\langle x\rangle_{0}$. This is due to the distribution of the magnetic field values along the hourly interval, and to the fact that, with a continuous gap, the central minutes are more likely to be extracted than those at both ends of the interval. This also implies that the RMS value evaluated from Eq. (1) (3.5 nT in our example) is slightly different from the standard deviation of the distribution of the differences $\langle x\rangle_{-20}^{i}-\langle x\rangle_{0}(3.1 \mathrm{nT})$, especially when dealing with long gaps. Despite this, in the interests of readability we will continue to refer to $\mathrm{RMS}_{-g} / \sigma$ as the relative standard uncertainty.

We are now interested in the distribution of the relative standard uncertainties $\mathrm{RMS}_{-g} / \sigma$ over different hourly intervals. Given a gap length $g$, is the $\mathrm{RMS}_{-g} / \sigma$ value constant regardless of the magnetic activity and observatory considered, as it was for the random case considered in the previous section? If it were, we would be able to construct a table similar to Table 3 and provide the typical accuracy of the mean estimated in the absence of $g$ continuous data points. Figure 6 shows the distribution of the $24 \mathrm{RMS}_{-g} / \sigma$ values corresponding to LIV M (moderate activity) for gap lengths $g=5,15,25$ and 40 .

The same experiment of Fig. 6 for the case of random extraction (Section 2.1) would have shown $\delta$-like distributions. Thus, for example, all the 24 points corresponding to $g=5$ would be clustered around $3.9 \%$, those for $g=15$ around $7.5 \%$, and so on (see Table 3). Unfortunately, this is not the case for the continuous extraction, so instead of a spot value, the data are now distributed in a finite interval; alternatively, we can understand this interval as reflecting the uncertainty of the parameter we are trying to evaluate, $\mathrm{RMS}_{-g} / \sigma$, which in turn is also an uncertainty. In our example, for a gap length $g=15, \mathrm{RMS}_{-g} / \sigma(\%)=25 \%$ $\pm 5 \%$ (where the \pm sign introduces the standard deviation 


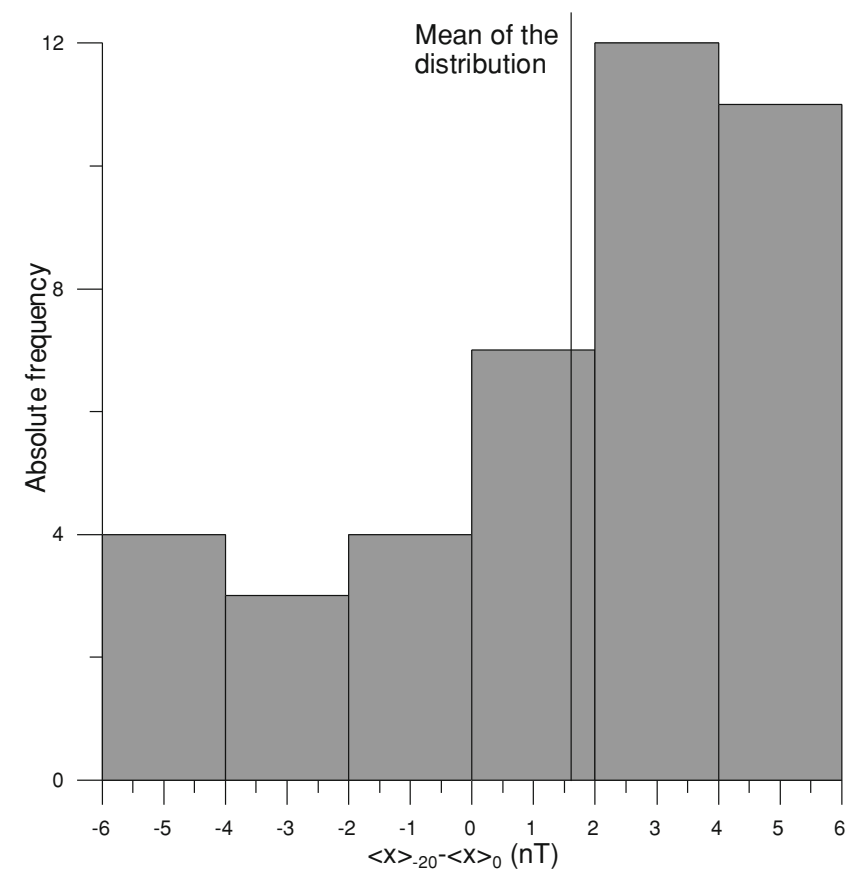

Fig. 5. Histogram showing the distribution of the 41 possible differences of the means after extraction of 20-minute-long gaps from the hourly interval 18:00-19:00 UT, January 9th, 2004, station LIV.

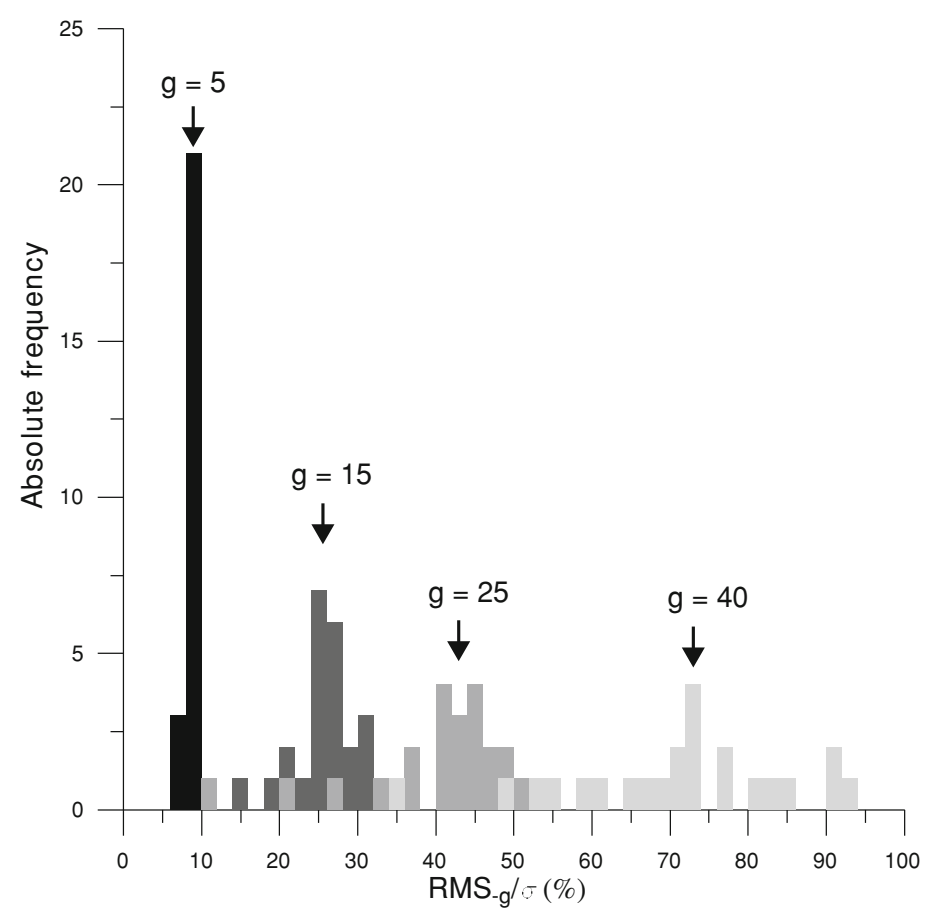

Fig. 6. Distribution of the individual $\mathrm{RMS}_{-g} / \sigma$ values $(g=5,15,25$ and 40$)$ for the 24 hourly intervals of LIV M (moderate magnetic activity). For a gap length $g=15$, for example, most of the 24 estimated means are between 20 and $30 \%$ of $\sigma$ away from the actual mean.

of the distribution associated to the relative standard uncertainty itself).

So far we have dealt with the particular case of LIV M, a mid-latitude observatory (as regards magnetic coordinates), but does the abovementioned interval increase with increasing latitude or activity level? Table 5 shows a poor or null dependence of the $\mathrm{RMS}_{-g} / \sigma$ interval on the observatory latitude.

In addition to this, Fig. 7 aims to show the dependence of the $\mathrm{RMS}_{-g} / \sigma$ values on the degree of magnetic activity, quantified here with the standard deviation $\sigma$ of the original hourly data.

The plots on Fig. 7 deserve special attention. The most apparent features are:

- The data distribution suggests a linear relationship between the relative standard uncertainty $\mathrm{RMS}_{-g} / \sigma$ and the logarithm of the standard deviation of the original 
Table 5. Dependence of the $\mathrm{RMS}_{-g} / \sigma(\%)$ interval on the observatory latitude. Observatories are arranged in order from higher to lower geomagnetic latitudes. For the computation of the $\mathrm{RMS}_{-g} / \sigma$ intervals in a given observatory, the three magnetic activity levels (A, M and Q) have been taken together.

\begin{tabular}{lccccc}
\hline Observatory (geom. lat.) & $g=5$ & $g=10$ & $g=15$ & $g=20$ & $g=25$ \\
\hline CMO $\left(65.4^{\circ} \mathrm{N}\right)$ & $8.0 \pm 0.8$ & $15 \pm 3$ & $22 \pm 5$ & $29 \pm 8$ & $35 \pm 10$ \\
LIV $\left(52.6^{\circ} \mathrm{S}\right)$ & $8.2 \pm 1.0$ & $16 \pm 3$ & $24 \pm 5$ & $32 \pm 8$ & $40 \pm 10$ \\
BOU $\left(48.4^{\circ} \mathrm{N}\right)$ & $8.2 \pm 0.7$ & $16 \pm 2$ & $23 \pm 5$ & $30 \pm 8$ & $38 \pm 10$ \\
EBR $\left(43.2^{\circ} \mathrm{N}\right)$ & $8.2 \pm 1.0$ & $16 \pm 3$ & $24 \pm 6$ & $32 \pm 8$ & $40 \pm 11$ \\
SJG $\left(28.3^{\circ} \mathrm{N}\right)$ & $8.2 \pm 0.8$ & $16 \pm 2$ & $24 \pm 4$ & $32 \pm 6$ & $40 \pm 8$ \\
\hline
\end{tabular}

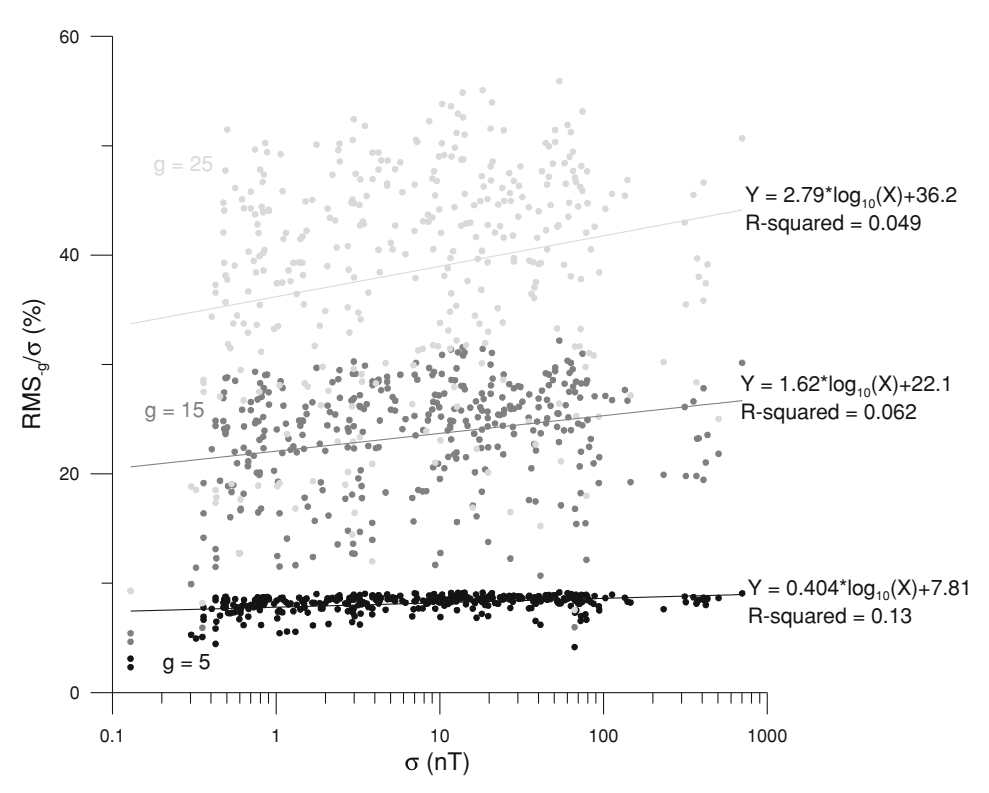

Fig. 7. Relative standard uncertainty, $\mathrm{RMS}_{-g} / \sigma$, as a function of $\sigma$ for the 360 hourly intervals after extraction of gap segments of length $g=5$, 15 and 25 .

data, $\log _{10}(\sigma)$, with the slope increasing with the gap length.

- Simultaneously, the noticeable scatter enhancement experienced for increasing gap lengths, $g$, greatly overshadows the $\sigma$ dependencies, resulting in small correlation coefficients ( $R$-squared in the figure).

- For a given gap length $g$, the scatter is slightly reduced with increasing $\sigma$.

- For a given gap length, the data are not symmetrically distributed around the mean, and the tail of the distribution is elongated towards low values of $\mathrm{RMS}_{-g} / \sigma$. On the contrary, an accumulation of data points is observed in the upper part of the distribution, which is more evident for small $g$ values.

In conclusion, $\sigma$ bears little influence on the relative standard uncertainty. This result is also important for our purpose of establishing an overall criterion irrespective of the activity level.

In summary, the data used to produce Fig. 7 reveals that, when a gap segment of 5 data points is present in an hourly interval, the 'standard error'" of the mean computed with the available data falls in the interval $0.023 \sigma-0.092 \sigma$ (minimum and maximum $\mathrm{RMS}_{-5}$ values of the total 360 test hourly intervals), with an important part of the probability $(68 \%)$ ranging between $0.076 \sigma$ and $0.088 \sigma$. This limited interval allows us to place narrow bounds for the "stan- dard error' in this case. Similarly, a gap length of 10 minutes yields a 'standard error' ranging ( $68 \%$ probability) between $0.135 \sigma$ and $0.183 \sigma$. As observed, this interval increases with the gap length, losing its usefulness beyond, say, $g=25$. For $g=30$, for example, this $(68 \%)$ interval is $0.35 \sigma-0.58 \sigma$, which is much too wide to establish a reliable criterion. An important conclusion from Table 5 and Fig. 7 is that the exact value for the standard error in these hourly intervals depends on the particular distribution of the magnetic field values within each specific hour, rather than on the observatory latitude or activity level.

However, in addition to the relative standard uncertainty of the estimated mean, we are also interested in the relative uncertainty at the level of confidence of $95 \%, U_{-g}^{95} / \sigma$; i.e., the relative error which will hardly ever be surpassed. A similar set of results applies in this case, whereby Fig. 8 is equivalent to Fig. 7 for the 95th percentile.

Again, $U_{-g}^{95} / \sigma$ slightly increases with $\sigma$, although the scatter overshadows this increment, maintaining it as relatively insignificant. In this case, the data are even more scattered than those of Fig. 7. Table 6 shows the central $68 \%$ interval of the distribution of $U_{-g}^{95} / \sigma$ values for each gap length $g$ from 0 to 25 ( 5 by 5 ).

In order to illustrate the results, we can say we are reason-

\footnotetext{
${ }^{1}$ Although it is preferable to use 'standard uncertainty' for the reasons extensively put forward in the 'GUM' (ISO, 1993), at this point we use 'standard error' to ease reading.
} 


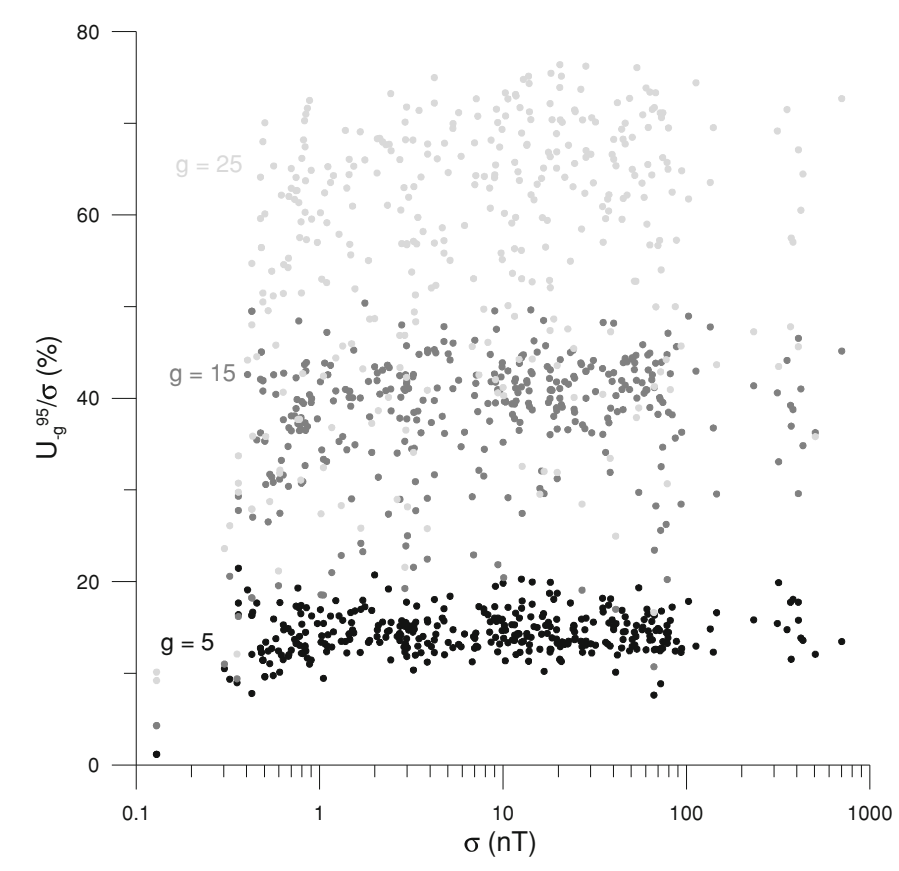

Fig. 8. Equivalent to Fig. 7 for the 95 th percentile case.

Table 6. Upper and lower limits of the central $68 \%$ accumulated probability for $U_{-g}^{95} / \sigma$. Values are percentages. Alternatively, this interval can be understood as the standard uncertainty interval associated to the parameter $U_{-g}^{95} / \sigma$.

\begin{tabular}{lcccccc}
\hline Gap length $g \rightarrow$ & 0 & 5 & 10 & 15 & 20 & 25 \\
\hline Upper and lower limit of the 68\% central interval & $0-0$ & $12-17$ & $23-31$ & $31-44$ & $37-57$ & $43-70$ \\
\hline
\end{tabular}

ably confident (at the $95 \%$ level) that the mean computed with an absence of 5 running minutes will not be off the actual mean by more than $0.14 \sigma \pm 0.02 \sigma$ (see Table 6 , column with header $g=5)$. The stated uncertainty $( \pm 0.02 \sigma)$ arises from the dependence of the $U_{-g}^{95} / \sigma$ on the distribution of the magnetic field values within the specific hourly interval considered, rather than on the observatory latitude or activity level itself, so that $68 \%$ of the analyzed hours (i.e., $0.68 \times 360=245$ ) have this $95 \%$ level within $0.02 \sigma$ around $0.14 \sigma$. Again, this differs from the case of random extraction, where we had spot values for $U_{-g}^{95} / \sigma$ instead of an interval.

The above results are based on analysing each individual hourly interval separately. The relative standard and $95 \%$ confidence level uncertainties are obtained for each hour, and the figures are based on the probability that a (new) hourly interval, with its particular number of missing data, has a certain value of uncertainty. Let us refer to it as the 'individual' approach. Nevertheless, we can go one step further by placing the statistics of all the stations and activity levels together, i.e., the 360 analyzed hourly intervals. We will refer to this as the 'simultaneous' approach so as to differentiate it from the previous one. To a certain extent, we believe this to be an appropriate and correct approach since we have shown that the observatory or magnetic activity in question bear little, if any, influence on the results. Thus the new procedure will be:

(1) Consider all the possible ways of extracting $g$ running minutes from an individual hourly interval and com- pute the different means, $\langle x\rangle_{-g}^{h, i}$, where the $h$ index stands for the specific hourly interval and $i$ for a particular extraction combination.

(2) Divide the differences between the estimated and actual means by $\sigma$ (of that particular hour), so that the relative differences $\left(\langle x\rangle_{-g}^{h, i}-\langle x\rangle_{0}^{h}\right) / \sigma^{h}$ are obtained.

(3) Repeat this process for the 360 hours ( $h=1$ to 360 ).

(4) Finally, we put all these relative differences together.

Thus, for a gap length $g=5$, a total of $360 \times(61-5)=$ 20160 relative differences are obtained, see Fig. 9. It is then straightforward to obtain the 95th percentile of this distribution. As well as this, the $\mathrm{RMS}_{-g} / \sigma$ value is obtained from Eq. (1) replacing $\left(\langle x\rangle_{-g}^{i}-\langle x\rangle_{0}\right)$ with $\left(\langle x\rangle_{-g}^{h, i}-\langle x\rangle_{0}^{h}\right) / \sigma^{h}$. The results are summarized in Table 7 and Figs. 10 and 11.

As seen in Fig. 10 for the relative standard uncertainty, except for $g=59$, the results of both methods (solid and discontinuous lines) roughly coincide. The solid line in the right-hand part of Fig. 10 is a linear (through the origin) fit of the relative standard uncertainty obtained with the simultaneous analysis method for small and moderate gap lengths. The empirical relationship between both magnitudes is found to be:

$$
\frac{\mathrm{RMS}_{-g}}{\sigma} \approx 0.0158 g
$$

The results from both methods are not so close for the case of $U_{-g}^{95} / \sigma$ (Fig. 11), especially for $g>15$. The solid line in the right-hand part of Fig. 11 is a quadratic (through the origin) fit of $U_{-g}^{95} / \sigma$ as obtained by the simultaneous ap- 


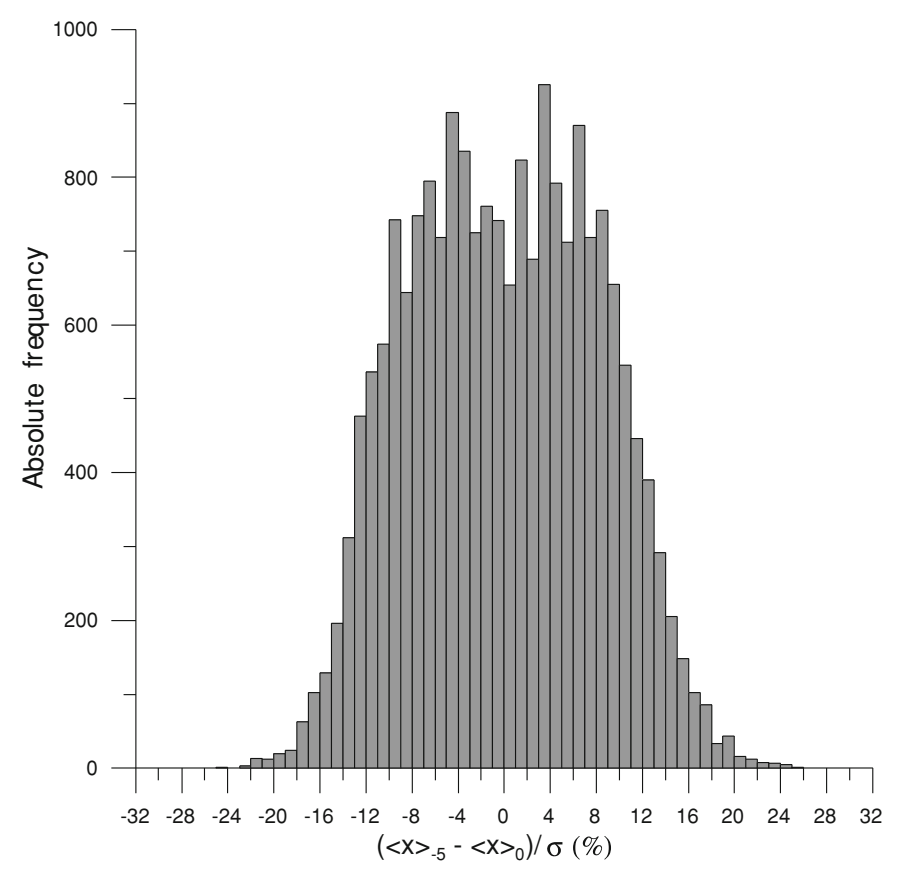

Fig. 9. Distribution of the 20160 relative differences obtained as a result of a simultaneous analysis for the whole set of hourly intervals for a gap length $g=5$.

Table 7. $\mathrm{RMS}_{-g} / \sigma$ and $U_{-g}^{95} / \sigma$ of the overall distribution of the relative differences for the 360 hourly intervals being studied after extraction of gap segments of different lengths (simultaneous analysis). Values are percentages.

\begin{tabular}{lllllll}
\hline Gap length $g \rightarrow$ & 0 & 5 & 10 & 15 & 20 & 25 \\
\hline RMS $_{-g} / \sigma$ of the global set & 0 & 8.1 & 16 & 24 & 31 & 39 \\
$U_{-g}^{95} / \sigma$ of the global set & 0 & 14 & 28 & 41 & 54 & 67 \\
\hline
\end{tabular}

proach for small and moderate gap lengths. The empirical relationship between both magnitudes is:

$$
\frac{U_{-g}^{95}}{\sigma} \approx 0.0289 g-0.0000942 g^{2}
$$

Thus, for an hourly interval with a continuous gap segment of 6 minutes in length, which means $10 \%$ of the data is missing, the estimated mean will have a relative standard uncertainty of the order of $9 \%$ (result from Eq. (2)). Furthermore, we can be confident of our mean within a relative uncertainty of the order of $17 \%$ (result from Eq. (3)).

Finally, it may be of interest to note that a value for both the absolute root mean square, RMS, and 95\% confidence limit, $U^{95}$, may be obtained by multiplying Eqs. (2) and (3) by $\sigma$. Of course, we do not know the exact value of this parameter when dealing with missing data (since it is defined for the complete data set), but to a first approximation we can evaluate $\sigma$ with the available data. Furthermore, it is worth mentioning that it is probably meaningless to obtain a mean with an accuracy higher than the resolution the MHVs will finally be reported with. In this sense, care must be taken when obtaining $U^{95}$ via Eq. (3) when dealing with extremely quiet intervals.

\subsection{Comparison between the random and continuous extraction approaches}

A direct comparison of Tables 3 and 4 with Table 7 shows that the random extraction method is not appropriate for outlining the results of the MHV problem, since data are unlikely to be missing in a randomly distributed way over a real hourly interval. One continuous gap is, by far, the most common way that minute values are absent. Cases with 2 gaps are the second most common situation (Schott and Linthe, 2007). A study of the number of gap segments per hour would yield slightly different results in each observatory. Nevertheless, we can take the results for the EBR observatory as an orientation. The results show that a 1 gap segment (i.e., one continuous gap) has a $94 \%$ probability, while 2 gap segments account for virtually all the remaining $6 \%$ of cases. Thus, in a real situation the results for the uncertainties will be somewhere between those obtained in the two preceding subsections. In the following part we will try to find out how far this $6 \%$ can affect the results given in Section 2.2.

It is worth mentioning that, as expected, the uncertainty associated with a single gap is much greater than the uncertainty of randomly distributed gaps; thus, for a given number of missing data, the estimated mean will be closer to the actual one in the latter case. Let us suppose that the assumption stated at the beginning of Section 2 is a general rule: for a fixed number of missing data, long gaps (i.e., fewer segments) are less reliable than short gaps (greater number of segments) when considering the mean computation.

Let us now consider the 'simultaneous' approach taken at the end of Section 2.2, for which the 20160 relative differences for $g=5$ are shown in Fig. 9. These correspond to the case of one gap, so when introducing the rela- 

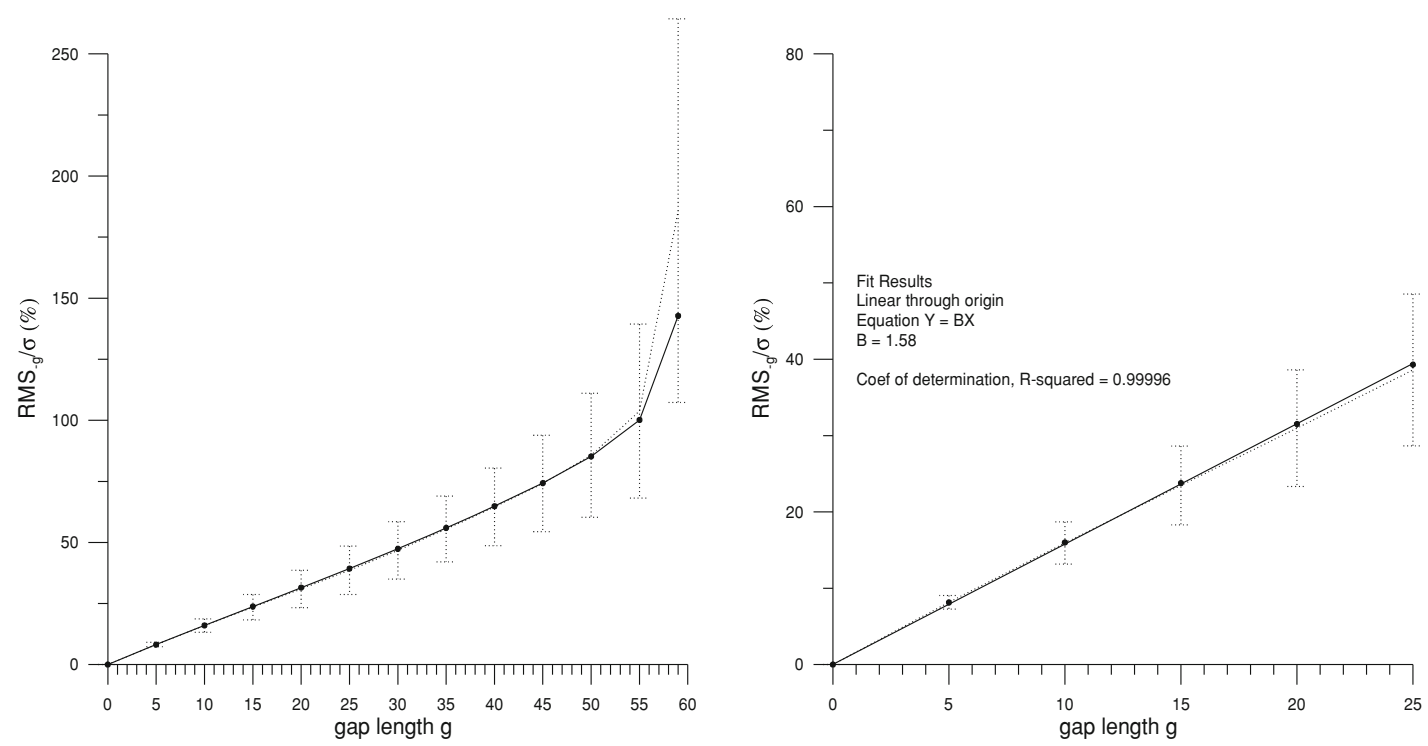

Fig. 10. The left-hand graph shows the standard uncertainty of the relative differences after extraction of different gap lengths $g$, whereas the right-hand plot shows a zoom for small and moderate $g$ values. Discontinuous lines show the distribution of the relative standard uncertainties and error bars on them bound the central $68 \%$ of analyzed hours (results from the 'individual' analysis); solid lines correspond to the standard uncertainty determination of the overall distribution of the relative differences (results from the 'simultaneous' analysis).
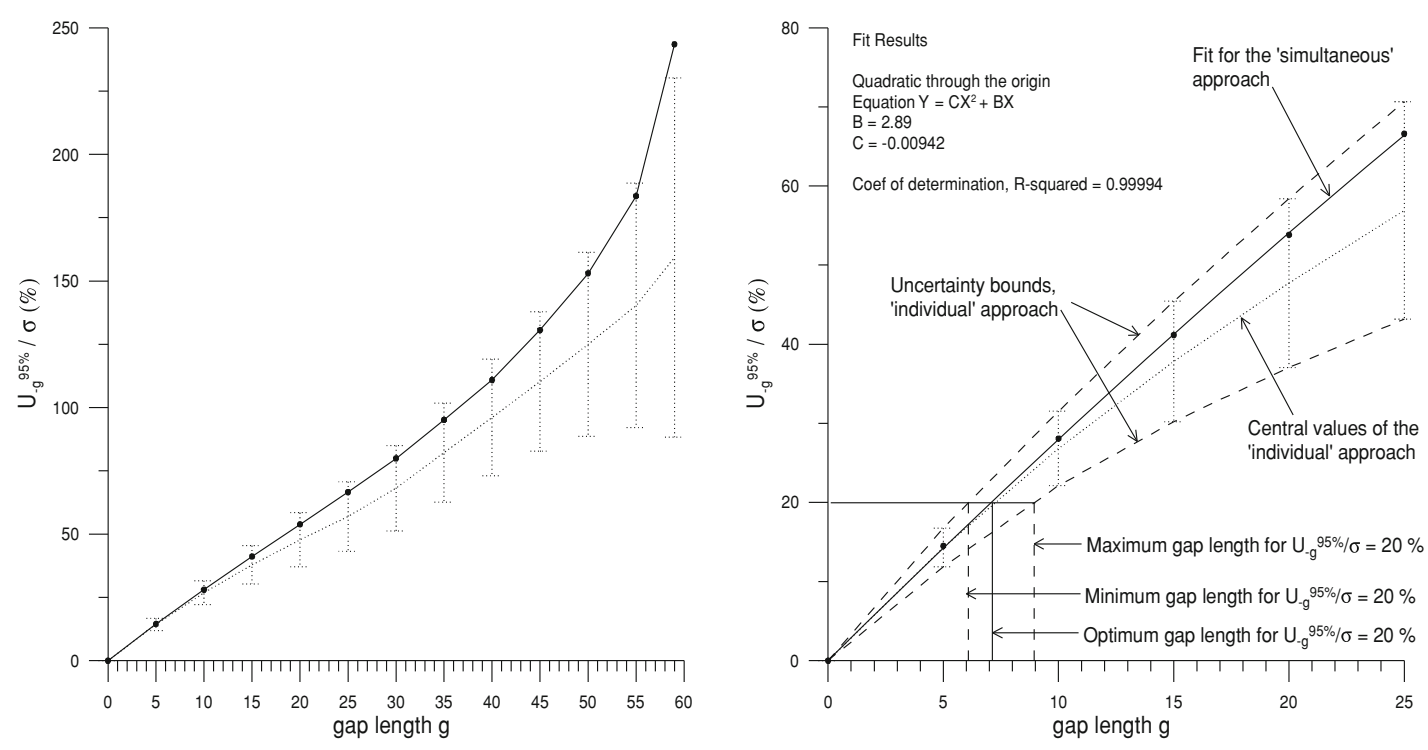

Fig. 11. Same as Fig. 10 for the relative $95 \%$ confidence level. Uncertainty bounds have been placed around the 'individual' approach central values, so as to include the $68 \%$ of the analyzed hourly intervals (i.e., 245). As an example, the correspondence between a particular value of $U_{-g}^{95}$ (20\% of $\sigma$ ) and its associated gap length (between 6 and 9 , with a maximum probability corresponding to 7 ) is outlined.

Table 8. Maximum percentage reduction of the 95th percentile for a realistic gap segment distribution with respect to the 95th percentile obtained when considering only continuous gaps (Table 7).

\begin{tabular}{lccccccccccccc}
\hline Missing data $g$ & 0 & 5 & 10 & 15 & 20 & 25 & 30 & 35 & 40 & 45 & 50 & 55 & 59 \\
\hline$\%$ reduction & 0 & 0.2 & 0.2 & 0.2 & 0.3 & 0.3 & 0.4 & 0.6 & 0.7 & 0.9 & 1.6 & 1.7 & 0.7 \\
\hline
\end{tabular}

tive differences obtained with 2 (or more) gap segments in the proportion $6 / 94$ (= probability of 2 or more gap segments over 1 gap segment), the number will increase to $20160 \times 100 / 94 \approx 21447$. The new 1288 added points will be distributed in the histogram of Fig. 9. However, if we make the assumption stated in the previous paragraph, the corresponding relative differences will be closer to 0 than those of the continuous case, so they will accumulate in the central part of the histogram. Computing the 95th percentile of the new distribution involves rejecting the $5 \%$ of the cases corresponding to the greatest relative differences, which in the worst case will not affect the added points. This means we must reject $0.05 \times 21447=1072$ points of the former distribution tail, which corresponds to retaining $94.68 \%$ of the original 20160 points. In summary, the lower limit for the real 95th percentile (i.e., considering both one 
and more than one gap segments in adequate proportions) is equivalent to applying the 94.68th percentile to the distribution of the continuous extraction case; obviously, the upper limit constitutes the former 95 th percentile. Based on this, Table 8 summarizes the maximum reductions with respect to the last row of Table 7 , assuming that the proportion $6 / 94$ is maintained for each number of missing data, $g$.

As seen, the reductions are imperceptible in most cases. The same can be seen even when the proportion of 2 or more gap segments is considerably greater than $6 \%$, so we can take the results obtained for the continuous case (Table 7) as sufficiently good approximations, rather than those given in Tables 3 and 4.

\section{Conclusions and Future Work}

The degree of accuracy of the MHVs is related to the (natural) magnetic variability of the respective hour. Thus, rather than using a fixed parameter, in our discussion we have referred to the standard deviation of the original data.

Our analysis set out to provide a general answer to the penultimate question posed in the introduction: When computing an MHV, what is the maximum number of missing data we can permit in an hourly interval, and still be reasonably confident that the (pre-established) required relative accuracy is still achieved? The answer to this question is not a simple figure and needs qualification. We analyzed a total of 360 hourly intervals from observatories at different latitudes and diverse magnetic activity levels, and we concluded that the answer depends principally on the particular distribution of the magnetic field values within the hourly interval, rather than on a specific observatory or magnetic activity at that time. This important fact allowed us to establish a general rule roughly valid for any location and activity level; nevertheless, the mentioned dependence on a given distribution of the minute values within the hour gives rise to a certain 'range of possible answers' to the question posed above. In this sense, the uncertainty (corresponding to a confidence level of $95 \%$ ) of the mean of our hourly interval in question, with a given amount of missing data, may fall within a finite interval. As the number of missing data increases $(g>25)$ the answer to our question becomes increasingly vague, since the analyzed hours show increasingly disparate outcomes, or equivalently, the referred interval is too large for a practical purpose. However, the results presented for shorter gaps are quite consistent, and at least we have an order of magnitude for the uncertainty associated to greater gaps

In this paper we provide some useful tools relating relative accuracy and number of missing data. Thus, once a data user has established their required relative accuracy, our procedure provides a range for the maximum number of missing data to be permitted in the MHVs of their study. Let us consider the example of a data user requiring an error in the estimated mean of, at most, $20 \%$ of the standard deviation of the original data (i.e., $0.2 \sigma$ ). From our analysis, it is probable to obtain this result with a maximum number of missing data ranging from 6 to 9 minutes (this stems from the uncertainty bounds displayed in Fig. 11), depending, again, on variables which cannot be controlled a priori, such as the particular distribution of the minute values in each specific hourly interval. Given that the maximum probability in this case is reached near 7 (Eq. (3) or Fig. 11 again), we suggest this figure as the optimum maximum tolerable number of lost data in the hourly intervals of the analysis. In this way, it is easy to implement a simple algorithm which rejects hourly intervals with less than 53 minutes of data, ensuring (at the level of confidence of 95\%) that its MHVs will not be off the true mean by more than 0.2 standard deviations of the original data set. As an alternative to a self-computed threshold for each particular MHV user, if a general consensus is attained as regards the 'average' relative accuracy required by data users, the IAGA association can establish a maximum number of missing data in the hourly intervals for computation of the observatories' MHVs. Furthermore, as suggested in the last IAGA Workshop, it would even be possible to report an estimation of the standard uncertainty of each computed MHV; this would be achieved by multiplying Eq. (2) by the $\sigma$ value obtained with the available data in each hourly interval.

Although $X$ and $H$ are the most widely-used magnetic elements in modelling and magnetic field indexing, we encourage the undertaking of an analysis for $Y$ and $Z$ as well, although similar results are expected $a$ priori.

Acknowledgments. The authors wish to thank the USGS for supplying the data from College, Boulder and San Juan observatories. They are also very grateful to Dr. L. F. Alberca, for his fruitful comments and suggestions. This work has been supported by Spanish project CGL2006-12437-C02-02/ANT of the Ministerio de Ciencia e Innovación.

\section{References}

Green, P., Lunar and solar daily variations of the geomagnetic field at Toolangi, Pure Appl. Geophys., 101(1), 194-204, 1972.

ISO, Guide to the Expression of Uncertainty in Measurement, Geneva, Switzerland: International Organization for Standardization, 1993.

Le Mouël, J., V. Kossobokov, and V. Courtillot, On long-term variations of simple geomagnetic indices and slow changes in magnetospheric currents: The emergence of anthropogenic global warming after 1990?, Earth Planet. Sci. Lett., 232(3-4), 273-286, 2005.

Mandea, M., 60, 59, 58, ... How many minutes for a reliable hourly mean?, Proceedings of the Xth IAGA Workshop, Hermanus, 112-120, 2002.

Martini, D. and K. Mursula, Correcting the geomagnetic IHV index of the Eskdalemuir observatory, Ann. Geophys., 24(12), 3411-3419, 2006.

Rangarajan, G. K., Some features of annual variation in the equatorial geomagnetic field, Indian J. Radio Space Phys., 11, 152-155, 1982.

Schott, J. J. and H. J. Linthe, The hourly mean computation problem revisited, Proceedings of the XIIth IAGA Workshop, Belsk, 135-143, 2007

Svalgaard, L. and E. W. Cliver, Interhourly variability index of geomagnetic activity and its use in deriving the long-term variation of solar wind speed, J. Geophys. Res., 112(A10), A10111, 2007.

Taylor, J. R., An Introduction to Error Analysis. The study of Uncertainties in Physical Measurements, Oxford University Press, California, 1982.

Torta, J. M., J. J. Curto, and P. Bencze, Behaviour of the quiet day ionospheric current system in the European region, J. Geophys. Res., 102(A2), 2483-2494, 1997.

Torta, J. M., L. R. Gaya-Piqué, J. J. Curto, and D. Altadill, An inspection of the long-term behaviour of the range of the daily geomagnetic field variation from comprehensive modelling, J. Sol. Atmos. Terr. Phys., doi: 101016/j.jastp.2008.06.006, 2008.

Walker, J. K., V. Y. Semenov, and T. L. Hansen, Synoptic models of high latitude magnetic activity and equivalent ionospheric and induced currents, J. Atmos. Terr. Phys., 59, 1435-1452, 1997.

S. Marsal and J. J. Curto (e-mail: jjcurto@obsebre.es) 